\title{
FFT-Based Analysis of Periodic Structures in Microacoustic Devices
}

\author{
Bernhard Jakoby, Senior Member, IEEE, and Michael J. Vellekoop
}

\begin{abstract}
Periodic structures utilized as transducer or reflector elements play an important role in microacoustic wave devices. Such structures can be described using approximate analytical models. However, to obtain the accuracy required for reliable device simulation, numerical methods have to be employed. In this contribution, we present an efficient numerical approach to calculate the dispersion curves associated with microacoustic modes propagating in periodic structures; the method is demonstrated for the case of Love wave modes. The computational efficiency is related to the utilization of the FFT algorithm in a hybrid Method of Moments (MoM) /Mode-Matching analysis. From the obtained dispersion curves, characteristic parameters such as the stopband width can be obtained which can be used in a coupling-of-modes (COM) model of the structure.
\end{abstract}

\section{INTRODUCTION}

I N THE ANALYSIS of periodic structures, so-called Floquet modes are frequently encountered [1]. In a wave guiding structure with periodic perturbations in the propagation direction $(x)$, eigenmodes can be obtained in "Floquet-periodic" form:

$$
\psi(x)=\psi_{p}(x) \mathrm{e}^{-j k_{0} x}
$$

where $\psi(x)$ stands for any of the field components associated with the mode, and $\psi_{p}(x)$ denotes a periodic function obeying the same period $p$ as the perturbation: $\psi_{p}(x+p)=\psi_{p}(x) . k_{0}$ represents the characteristic wavenumber for the Floquet mode, which depends on the (angular) frequency $\omega$. Because of the periodic function $\psi_{p}(x)$, a Floquet mode does not show a purely harmonic $x$ dependence but represents a superposition of harmonic wave functions with wavenumbers $k_{n}=k_{0}+n 2 \pi / p$, where $n=-\infty, \ldots, \infty$ as it can be perceived by expanding $\psi_{p}(x)$ in a Fourier series. Waves in periodic microacoustic structures have been analyzed utilizing the MoM together with the concept of a Floquet periodic Green's function (see, e.g. [2]-[5]).

In this contribution, we present an approach employing a hybrid MoM/Mode-Matching technique that utilizes

Manuscript received June 9, 1998; accepted November 9, 1999. This work was supported by the Brite-Euram project BE-951745:MIMICS.

The authors are with Delft University of Technology, Electronic Instrumentation Lab./DIMES, Mekelweg 4, $2628 \mathrm{CD}$ Delft, The Netherlands (e-mail: bernhard.jakoby@de.bosch.com).

B. Jakoby's new address: Robert Bosch GmbH, K8/SPP5, Tübinger Strasse 123, 72762 Reutlingen, Germany.

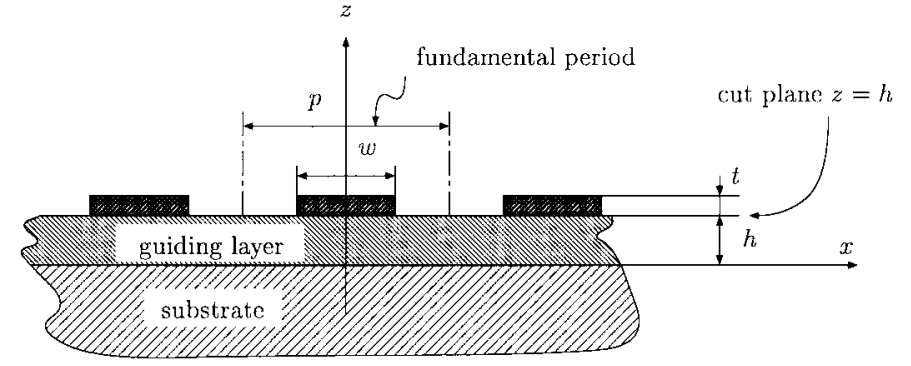

Fig. 1. Love wave guiding structures with periodic perturbations.

the FFT algorithm to obtain a computationally efficient method.

\section{THEORY}

To illustrate the procedure, we outline the analysis method by considering Love waves propagating in a waveguide that is periodically loaded with elastic blocks (Fig. 1). Love waves are of interest for liquid sensor applications because they are shear polarized and do not couple with compressional acoustic wave modes in adjacent liquids [6], [7]. The structure is two-dimensional, showing no variations in the $y$ direction; hence, $\partial / \partial y \equiv 0$. This geometry can also be interpreted as a model for reflector structures on a Love wave device. For the sake of simplicity, we demonstrate the approach for Love waves propagating on a nonpiezoelectric, isotropic substrate. (The generalization to more general cases will be illustrated later.)

For the following, we assume harmonic time dependence of all field quantities and suppress the common factor $\exp (j \omega t)$. We consider purely shear polarized Love modes for which the only non-vanishing displacement component is the displacement in $y$ direction, $u_{y}$. Because $u_{x}=u_{z}=$ 0 and $\partial / \partial y \equiv 0$, the only non-vanishing elements of the stress tensor $T_{i j}[8]$ are $T_{x y}\left(=T_{y x}\right)$ and $T_{y z}\left(=T_{z y}\right)$. Because of the isotropy of the materials and because all material boundary planes are orthogonal to the $x z$ plane, no coupling to differently polarized (sagittal) modes occurs. The interaction between the Love wave and the perturbing blocks occurs in the plane $z=h$. The boundary conditions in the fundamental period $[-p / 2, p / 2]$ are

$$
\left.\left.\begin{array}{r}
\left.\left.T_{y z}\right|_{z=h}=0 \text { for }|x| \in\right] w / 2, p / 2[, \\
\left.T_{y z}\right|_{z=h^{+}}=T_{y z},\left.\right|_{z=h^{-}} \\
\left.u_{y}\right|_{z=h^{+}}=\left.u_{y}\right|_{z=h^{-}}
\end{array}\right\} \text {for } x \in\right]-w / 2, w / 2[,
$$

which, accordingly shifted, also holds in all other periods. 


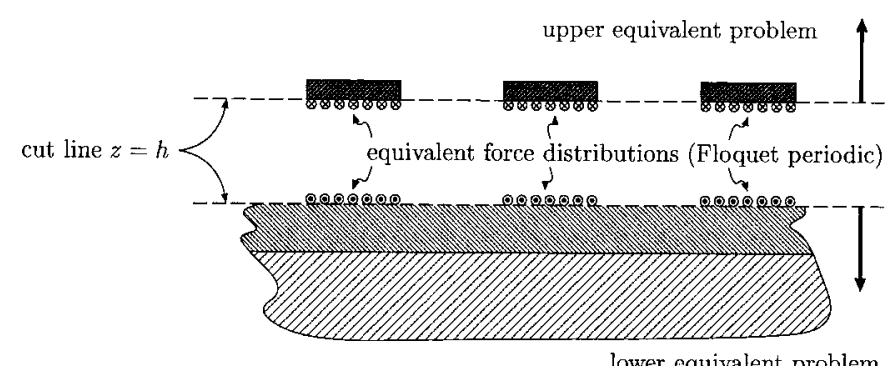

Fig. 2. Splitting the structure in two equivalent problems. Equivalent forces in the split plane represent the mechanical interaction between the two problems.

The regions $|x| \in] w / 2, p / 2[$ and $x \in]-w / 2, w / 2[$ characterize the free and the block-covered regions in the fundamental period, respectively.

For the analysis, we virtually cut the structure along $z=h$ to obtain two equivalent problems, where the interaction between the two is taken into account by oppositely directed but equally strong, $\pm y$ directed, "equivalent" surface force distributions in the block-covered regions of the cut planes (Fig. 2). These force distributions prescribe equal $T_{y z}$ distributions in the cut plane of the two equivalent problems [in accordance with (3)]. The $u_{y}$ distributions resulting from the impressed $T_{y z}$ distributions in the two equivalent problems have to be equal in the block-covered regions, which completes the boundary condition (3).

\section{A. Equivalent Problem for the Lower Half Space}

The equivalent problem for the lower half space $z<h$ corresponds to the unloaded Love-wave waveguide excited by an impressed surface force distribution. Because the considered fields are Floquet-periodic, we can write the displacement $u_{y}(x)$ because of an impressed $T_{y z}$ distribution in the cut plane $z=h$ as a convolution of a Floquet periodic Green's function $G_{p}\left(x, k_{0}\right)$ [2], [3], [5] with the stress distribution in the fundamental period

$$
u_{y}(x)=\int_{-\infty}^{+\infty} G_{p}\left(x-x^{\prime}, k_{0}\right) T_{y z}^{0}\left(x^{\prime}\right) d x^{\prime} .
$$

Here, and in the following, the superscript 0 indicates quantities in the fundamental period, and, for the sake of notational simplicity we use the convention that field quantities $u_{y}$ and $T_{y z}$, are considered in the cut plane, $z=h$ if only one argument $(x)$ is provided. For $T_{y z}^{0}$ we thus have $T_{y z}^{0}(x)=0$ for $|x|>w / 2$. The relationship with the $T_{y z}$ distribution in the entire cut plane is given by

$$
T_{y z}(x)=\sum_{n=-\infty}^{\infty} T_{y z}^{0}(x-n p) \exp \left(-j n k_{0} p\right) .
$$

The Floquet-periodic Green's function $G_{p}\left(x, k_{0}\right)$ is defined as the displacement $u_{y}$ (in $z=h$ ) generated by a periodic array of Floquet-periodic line forces creating a stress distribution $T_{y z}(x)=\sum_{n} \delta(x-n p) \exp \left(-j n k_{0} p\right)$ in the plane $z=h$ of the unloaded structure $[\delta(x)$ denotes the Dirac delta function]. Because of the application of $G_{p}$, the resulting $u_{y}(x)$ includes the effects of the entire impressed stress distribution $T_{y z}$, where the boundary condition (2) has been considered as well.

\section{B. Equivalent Problem for the Upper Half Space}

In this case, a simple analytical solution for the fields in the block can be found in terms of a modal expansion. The fields of blocks in different periods are not coupled with each other, and, hence, it is sufficient to consider the fields in the fundamental period $\left(T_{y z}^{0}\right.$ and $\left.u_{y}^{0}\right)$. We utilize the following modal functions for $u_{y}(n=0,1,2, \ldots)$ :

$$
u_{y, n}(x, z) \propto \cos \left(k_{z, n}(z-h-t)\right) m_{n}(x),
$$

where

$$
\begin{aligned}
m_{n}(x) & =\left\{\begin{array}{l}
\cos \left(x \frac{n \pi}{w}\right), \mathrm{n}=\text { even } \\
\sin \left(x \frac{n \pi}{w}\right), \mathrm{n}=\text { odd }
\end{array}\right. \\
k_{z, n} & =\sqrt{\frac{\omega^{2} \rho_{b}}{\mu_{b}}-\left(\frac{n \pi}{w}\right)^{2}}
\end{aligned}
$$

Here, $\mu_{b}$ and $\rho_{b}$ denote the shear modulus and the mass density of the block material, respectively. It can readily be verified that the corresponding stress tensor elements given by $[8]$

$$
T_{y z}=\mu_{b} \frac{\partial u_{y}}{\partial z}, T_{x y}=\mu_{b} \frac{\partial u_{y}}{\partial x}
$$

fulfill the boundary conditions $T_{y z}=0$ and $T_{x y}=0$ at the block boundaries $z=h+t$ and $x= \pm w / 2$, respectively. Using these modes, we can set up an expansion for $T_{y z}^{0}$ and $u_{y}^{0}$ in the cut plane $z=h$ :

$$
\begin{aligned}
\left.T_{y z}^{0}(x)\right|_{z=h} & =\sum_{n=0}^{\infty} \tau_{n} m_{n}(x) \\
\left.u_{y}^{0}(x)\right|_{z=h} & =\sum_{n=0}^{\infty} \tau_{n} Z_{n} m_{n}(x)
\end{aligned}
$$

$\tau_{n}$ are the expansion coefficients and

$$
Z_{n}=\frac{\cot \left(k_{z, n} t\right)}{\mu_{b} k_{z, n}}
$$

denotes the acoustic modal impedance giving the ratio $u_{y} / T_{y z}$ at $z=h$ for mode $n$. Note that $k_{z, n}$ becomes imaginary from a certain value $n$ onward; in this case, the trigonometric functions turn into the corresponding hyperbolic functions, and $Z_{n}$ becomes purely imaginary. Truncating the previously mentioned modal expansion series would yield an approximation corresponding to a neglect of higher order modes. 


\section{Joining the Equivalent Problems}

To join the field solutions, we have to match the $T_{y x}$ distributions and the related $u_{y}$ distributions in the cut plane, which formally corresponds to a problem in an infinite dimensional function space. To make the problem numerically accessible, we utilize a MoM approach and reduce the dimensionality of the function space by introducing a suitable approximation. This is done by expanding $T_{y z}^{0}$ into a finite sum of $N$ rectangular pulse functions $P(x)$

$$
T_{y z}^{0}(x)=\sum_{n=1}^{N} \alpha_{n} P\left(x-x_{n}\right)
$$

where

$$
P(x)=\left\{\begin{array}{l}
1,|x|<w /(2 N) \\
0, \text { elsewhere }
\end{array}\right.
$$

and

$$
x_{n}=-w / 2+(n-1 / 2)(w / N)
$$

stands for the center point of pulse function number $n$. $\alpha_{n}$ are the expansion coefficients to be determined. The relationship of $\alpha_{n}$ with the expansion coefficients $\tau_{n}$ in (6) can simply be obtained by equating the right hand sides of (8) and (6), multiplying the resulting equation by

$P\left(x-x_{m}\right)$, and integrating with respect to $x$, which yields (using the orthogonality of the pulse functions $P(x-$ $\left.x_{m}\right)$ )

$$
\frac{w}{N} \alpha_{m}=A_{m n} \tau_{n}
$$

with

$$
A_{m n}=\int_{-\infty}^{+\infty} P\left(x-x_{m}\right) m_{n}(x) d x
$$

Here and subsequently, we use Einstein's summation convention, which means that we sum over doubly occurring indices. The translation coefficients $A_{m n}$ can be readily evaluated in closed form. It is near at hand to also truncate the modal expansion series (6) and (7) at $n=N-1$, which makes the mapping (10) unique and invertible $\left[A_{m n}\right.$ then corresponds to a square matrix].

For matching the corresponding $u_{y}$ distributions, we use a similar approach: equating the right-hand sides of (4) and (7), multiplying the so obtained equation with $P\left(x-x_{m}\right)$, and integrating with respect to $x$, we obtain

$$
B_{m n} \alpha_{n}=A_{m n} Z_{n} \tau_{n}
$$

with

$B_{m n}=\int_{-\infty}^{+\infty} P\left(x-x_{m}\right) \int_{-\infty}^{+\infty} G_{p}\left(x-x^{\prime}, k_{0}\right) P\left(x-x_{n}\right) d x^{\prime} d x$
We again note that it is sufficient to perform this matching in the fundamental period because we imposed Floquetperiodic fields by using $G_{p}$ in (4). Once the matrix elements $A_{m n}$ and $B_{m n}$ have been determined, a homogeneous matrix equation can be derived from (10) and (11) by eliminating $\alpha_{n}$ :

$$
\left(A_{m n}-\frac{N}{w} C_{m k} A_{k n} Z_{n}\right) \tau_{n}=0
$$

where $C$ is the inverse matrix of matrix $B$. To obtain a non-trivial solution for $\tau_{n}$, the determinant of the system matrix in (13) has to vanish, which yields a condition for the Floquet wavenumber $k_{0}$ for a prescribed $\omega$ (or vice versa). The numerical search for zeros of this determinant yields the dispersion curve $k_{0}(\omega)$ for the Floquet modes in the structure.

\section{Computational Procedure}

The computational efficiency of this approach is mainly determined by the evaluation of the matrix elements $B_{m n}$. The expression for $B_{m n}$ can be more conveniently calculated by introducing quantities in the spectral (Fourier) domain using the Fourier transform (spectral quantities are indicated by a tilde):

$$
\operatorname{FT}\{f(x)\}=\tilde{f}(\beta)=\int_{-\infty}^{+\infty} f(x) \exp (j \beta x) d x
$$

Using the convolution theorem and Parseval's theorem [9], we obtain

$$
B_{m n}=\frac{1}{2 \pi} \int_{-\infty}^{+\infty}|\tilde{P}(\beta)|^{2} \tilde{G}_{p}\left(\beta, k_{0}\right) \mathrm{e}^{-j \beta\left(x_{m}-x_{n}\right)} d \beta .
$$

The Floquet-periodic Green's function in spectral domain, $\tilde{G}_{p}\left(\beta, k_{0}\right)$, can be simply given in terms of the associated "ordinary" (i.e., non-periodic) spectral Green's function $\tilde{G}(\beta)$ being sampled by a train of Dirac delta functions ${ }^{1}$ $[2]:$

$$
\tilde{G}_{p}\left(\beta, k_{0}\right)=\tilde{G}(\beta) \frac{2 \pi}{p} \sum_{n=-\infty}^{\infty} \delta\left(\beta-k_{0}-n \frac{2 \pi}{p}\right)
$$

The (non-periodic) spectral Green's function for the unloaded waveguide can be obtained by a straightforward analysis (similar to the one used for spectral electric Green's functions [10]) as

$$
\tilde{G}(\beta)=\frac{1}{\beta_{l} \mu_{l}} \frac{\mu_{s} \alpha_{s} \tan \left(\beta_{l} h\right)+\mu_{l} \beta_{l}}{\mu_{s} \alpha_{s}-\mu_{l} \beta_{l} \tan \left(\beta_{l} h\right)}
$$

\footnotetext{
${ }^{1}$ The "ordinary" Green's function in spatial domain, $G(x)$, represents the fields $u_{y}(x)$ caused by a single, $y$-oriented unit line force at $x=0$ and $z=h$.
} 
where $\mu_{s}$ and $\mu_{l}$ denote the shear moduli of the substrate and the layer, respectively, and

$$
\beta_{l}=\sqrt{\frac{\omega^{2}}{v_{l}^{2}}-\beta^{2}}, \alpha_{s}=\left\{\begin{array}{c}
\sqrt{\beta^{2}-\frac{\omega^{2}}{v_{s}^{2}}}, \beta^{2}>\frac{\omega^{2}}{v_{s}^{2}} \\
j \sqrt{\frac{\omega^{2}}{v_{s}^{2}}-\beta^{2}}, \beta^{2}<\frac{\omega^{2}}{v_{s}^{2}}
\end{array} .\right.
$$

$v_{s}$ and $v_{l}$ denote the bulk shear velocities in the layer and the substrate material, respectively. Using (15) in (14) and considering the sampling property of the delta function, we finally arrive at

$$
B_{m n}=\frac{1}{p} \sum_{k=-\infty}^{+\infty}\left|\tilde{P}\left(\beta_{k}\right)\right|^{2} \tilde{G}\left(\beta_{k}\right) \mathrm{e}^{-j \beta_{k}\left(x_{m}-x_{n}\right)}
$$

where $\beta_{k}=k_{0}+k 2 \pi / p$. This series has to be truncated for numerical evaluation and can be effectively evaluated using an FFT if the number of considered series elements $K$ equals a power of two. We can truncate the series as (using the abbreviation $f_{k}=\left|\tilde{P}\left(\beta_{k}\right)\right|^{2} \tilde{G}\left(\beta_{k}\right)$ )

$$
\begin{array}{r}
B_{m n} \approx \mathrm{e}^{-j k_{0}\left(x_{m}-x_{n}\right)} \sum_{k=-K / 2}^{K / 2-1} f_{k} \mathrm{e}^{-j k \frac{2 \pi}{p}\left(x_{m}-x_{n}\right)}= \\
\mathrm{e}^{-j k_{0}\left(x_{m}-x_{n}\right)} \mathrm{e}^{j \pi \hat{x}} \sum_{k=0}^{K-1} f_{k-K / 2} \mathrm{e}^{-j \frac{2 \pi}{K} k \hat{x}}
\end{array}
$$

where the second sum representation has been obtained shifting the index variable $k$ and using the definition

$$
\hat{x}=\left(x_{m}-x_{n}\right) \frac{K}{p} .
$$

This second sum represents the discrete Fourier transform $f_{k-K / 2}$ provided that $\hat{x}$ is interpreted as a discrete variable. A discrete Fourier transform can be evaluated effectively using the FFT algorithm [11], which then yields the sum for discrete values $\hat{x}=0,1, \ldots, K-1$. Because of the periodicity property of the FFT, results for negative values of $\hat{x}$ are equal to those for $K-\hat{x}$. Hence, utilizing a single FFT run, we obtain approximations for $B_{m n}$ for a series of discrete $\left(x_{m}-x_{n}\right)$ values being equally spaced by $p / K$ [see (19)]. Depending on the geometrical ratio $w / p$, it is often possible to choose the number of expansion functions $N$ appropriately such that the actually needed spacing for the $\left(x_{m}-x_{n}\right)$ values, $w / N$, is an integer multiple of the spacing $p / K$ provided by the FFT result. If that is the case, the needed $B_{m n}$ elements are directly contained in the FFT output; otherwise, a simple interpolation procedure can be used to obtain the values for the desired relative distances $\left(x_{m}-x_{n}\right)$ from those that are delivered by the FFT $\left[B_{m n}\right.$ is a smooth function in $\left.\left(x_{m}-x_{n}\right)\right]$.

We remark that one could, in principle, also directly use the truncated modal series (6) and (7) as approximations for the interface distributions (without introducing the rectangular pulse approximation (8)). In that case, however, we could not evaluate the resulting $B_{m n}$ elements using the FFT approach because the shape of the

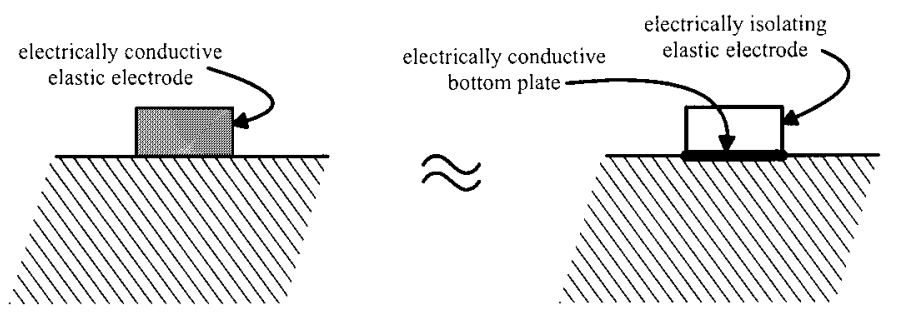

Fig. 3. Approximating a conducting electrode by a non-conductive one with conducting bottom.

expansion functions would vary and the used rectangular expansion functions differ only in their center position, which makes $B_{m n}$ a function of the relative center distance $\left(x_{m}-x_{n}\right)$ [see (14)].

Finally, we note that the electric interaction in case of a piezoelectric substrate and electrically conducting blocks (electrodes) can be taken into account by using a set of Green's functions including piezoelectricity and thus, taking electromechanic interaction into account also. The conductive electrode can be modeled effectively as an elastic block featuring a perfectly conducting bottom but being otherwise electrically isolating (Fig. 3). Thus, electric charges appear at the electrically conducting bottom of the electrode only. These charges can be expanded similar to the stress distribution, and the corresponding electric boundary condition is given by requiring constant electric potential on the electrode bottom. This model generally represents a very good approximation and is discussed in [4] where a combined MoM/Finite-Element method has been introduced. For non-conductive but dielectric blocks, the simplest approach would be to neglect the contribution of the electric perturbation introduced by the blocks, which also represents a reasonable approximation (especially for low-coupling substrates such as quartz).

\section{Numerical Results}

To illustrate the applicability of the approach, we calculated the dispersion diagram $k_{0}=k_{0}(\omega)$ for a waveguide with an ST quartz substrate that is covered with a fused quartz layer. The ST-quartz substrate is modeled by an isotropic material with a mass density $\rho_{s}=2650 \mathrm{~kg} / \mathrm{m}^{3}$ and a shear velocity $v_{s}=\sqrt{\mu_{s} / \rho_{s}}=5060 \mathrm{~m} / \mathrm{s}$. For the layer material, we have $\rho_{l}=2200 \mathrm{~kg} / \mathrm{m}^{3}, v_{l}=3766$ $\mathrm{m} / \mathrm{s}$. Calculations have been performed for gold $\left(\rho_{b}=\right.$ $\left.19300 \mathrm{~kg} / \mathrm{m}^{3}, v_{b}=1245 \mathrm{~m} / \mathrm{s}\right)$ and aluminum $\left(\rho_{b}=\right.$ $2700 \mathrm{~kg} / \mathrm{m}^{3}, v_{b}=3109 \mathrm{~m} / \mathrm{s}$ ) as block material.

Fig. 4 shows the dispersion characteristics for this structure. The width of the blocks was chosen such that they cover $1 / 8$ of the period: $w=0.125 p$. The dispersion curve for the free surface and curves for different relative block heights $t / p$ are shown in the vicinity of the boundary of the first Brillouin zone $k_{0}=\pi / p$ [1] where a characteristic stop band occurs.

The shift of the center of the stopband with respect to the free surface dispersion curve $\left(\Delta \omega_{c}\right)$ indicates the re- 


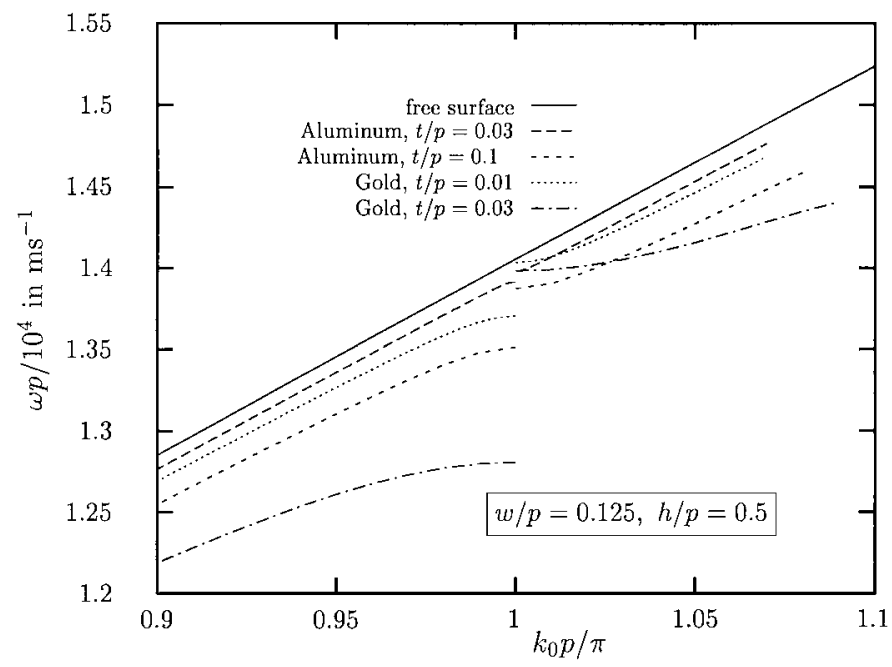

Fig. 4. Dispersion curves giving angular frequency $\omega$ vs the scaled Floquet wavenumber $k_{0} p / \pi$.

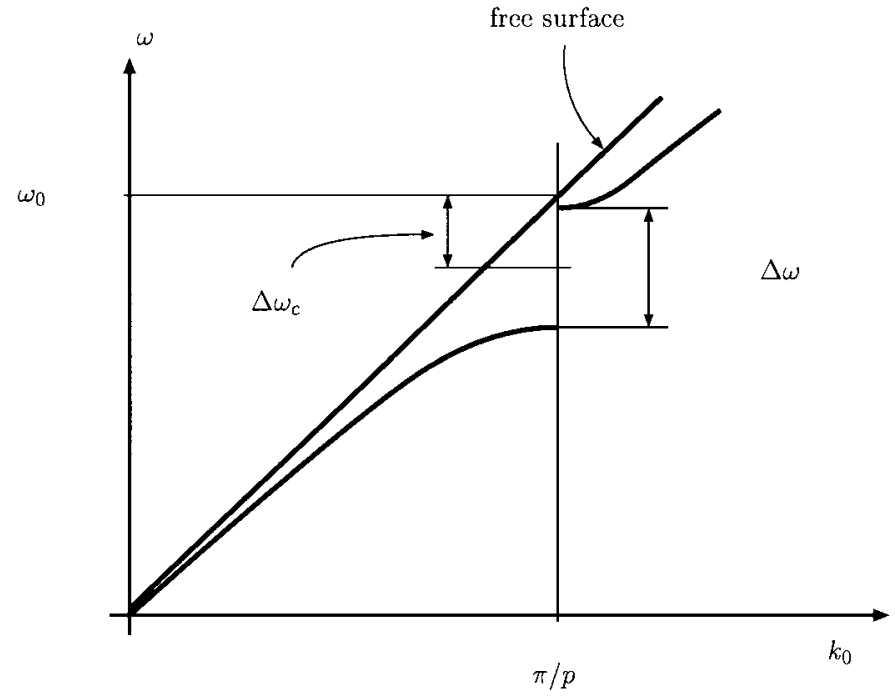

Fig. 5. Definition of band gap $\Delta \omega$ in the dispersion curve.

duction of the effective wave velocity compared with the unloaded case. The width of the stop band $\Delta \omega$ is a characteristic measure of the degree of the mechanical reflections caused by the blocks. These parameters can be used in a COM description of the periodic structure (see, e.g., [1], $[5],[12],[13])$. According to the COM theory, the expression $\pi \Delta \omega / 2 \omega_{0}$ can be interpreted as the reflection coefficient $\rho$ for a single block (Fig.Fig. 5). This value can be used in the design of reflector arrays; a periodic arrangement of $N$ reflecting strips yields a total reflection coefficient of $R=\tanh (N \rho)$ at the center frequency of the reflector [14].

It can be seen that, because of the much higher mass density, the gold blocks lead to larger band gaps compared with aluminum blocks. Note that the band gaps for gold and aluminum show about the same size for the cases $t / p=0.01$ for gold and $t / p=0.1$ for aluminum, which

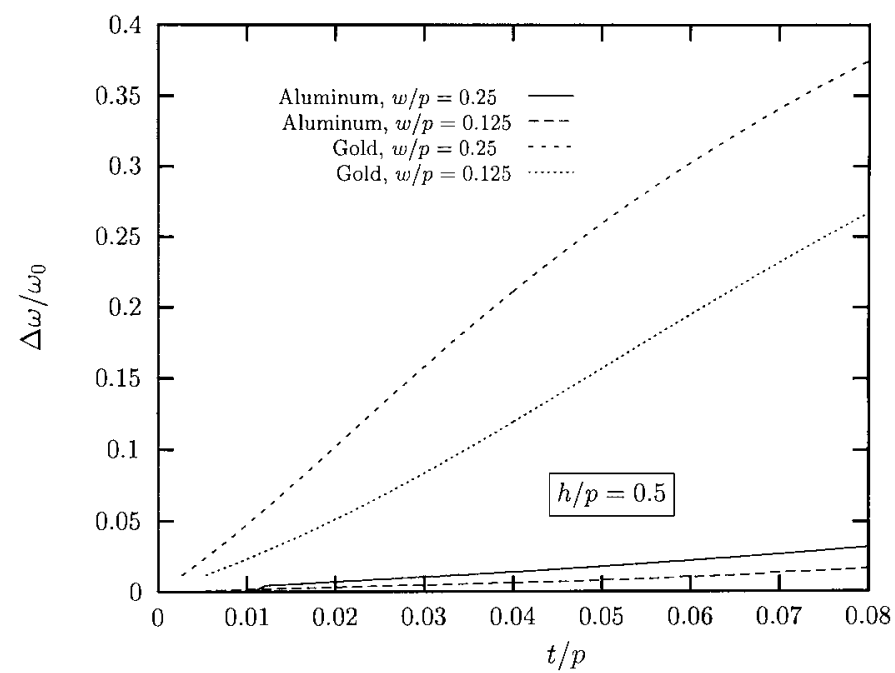

Fig. 6. Relative band gap $\Delta \omega / \omega_{0}$ vs relative block height $t / p$.

corresponds to a difference in height by a factor of ten. This value reflects the difference in the mass densities for the two materials. The upper branches of the curves stop at certain points. Above those end points, the mode becomes damped because of leaky wave excitation in the substrate [1].

Fig. 6 shows the relative band gap width for two different widths $w$ as a function of the block height $t$. We observe the effect that the band gap (corresponding to the reflection coefficient) increases for increasing electrode width. Again, the effect is roughly a factor ten greater for the gold blocks. Furthermore, the band gap increases for increasing block width, as can be seen by comparing the curves for different block widths.

\section{Conclusions}

We presented a numerical approach based on a hybrid MoM/Mode-Matching technique for the analysis of periodically loaded waveguides. The method has been outlined by considering the propagation of Love waves in a waveguide with periodic mass-loading. With appropriate modifications, the method is also applicable to other kinds of acoustic wave types. High computational efficiency has been achieved by utilizing the FFT for the calculation of the needed MoM interaction elements. As an example, we presented Brillouin dispersion diagrams that have been calculated for a sample structure. The results can be utilized to extract COM parameters for the structure.

\section{ACKnowledgments}

The authors thank Dr. Adrian Venema for many stimulating discussions and useful suggestions. 


\section{REFERENCES}

[1] C. Elachi, "Waves in active and passive periodic structures: A review," Proc. IEEE, vol. 64, pp. 1666-1698, Dec. 1976.

[2] C. Mecklenbräuker, "Piezoelektrische Wellenausbreitung in periodischen Metallisierungsstrukturen," M.S., Vienna Univ. Technol., Austria, 1992.

[3] A. R. Baghai-Wadji and C. Mecklenbräuker, "Propagation of piezoelectric waves in singly- and doubly-periodic metallic gratings," in Proc. 5th Eur. Freq. and Time Forum, Besançon, France, pp. 66-72, 1991.

[4] H. P. Reichinger and A. R. Baghai-Wadji, "Dynamic 2D analysis of SAW devices including massloading," in Proc. IEEE Ultrason. Symp., Tucson, AZ, pp. 7-10, 1992.

[5] V. P. Plessky and T. Thorvaldsson, "Periodic Green's functions analysis of saw and leaky saw propagation in a periodic system of electrodes on a piezoelectric crystal," IEEE Trans. Ultrason., Ferroelect., Freq. Contr., vol. 42, no. 2, pp. 280-293, 1995.

[6] G. Kovacs, G. W Lubking, M. J. Vellekoop, and A. Venema, "Love waves for (bio)chemical sensing in liquids," in Proc. IEEE Ultrason. Symp., Tucson, AZ, pp. 281-285, 1992.

[7] E. Gizeli, A. C Stevenson, N. J Goddard, and C. R Lowe, "A novel Love-plate acoustic sensor utilising polymer overlayers," IEEE Trans. Ultrason., Ferroelect., Freq. Contr., vol. 39, no. 5, pp. 657-659, 1992.

[8] B. A. Auld, Acoustic Fields in Solids. vol. I, New York: John Wiley \& Sons, 1973.

[9] G. Arfken, Mathematical Methods for Physicists. 2nd ed. New York: Academic Press, 1970.

[10] D. P. Morgan, Surface-Wave Devices in Signal Processing. Amsterdam: Elsevier, 1985.

[11] E. O. Brigham, The Fast Fourier Transform. Englewood Cliffs: NJ, 1974.

[12] H. A. Haus and P. V Wright, "The analysis of grating structures by coupling-of-modes theory," in Proc. IEEE Ultrason. Symp., Boston, MA, pp. 277-281, 1980.

[13] P. Bauerschmidt, R. Lerch, J. Machui, W. Ruile, and G. Visintini, "Reflection and transmission coefficients of SAW in a periodic grating computed by finite element analysis," in Proc. IEEE Ultrason. Symp., Honolulu, HI, pp. 421-423, 1990.

[14] P. S. Cross, "Properties of reflective arrays for surface acoustic resonators," in IEEE Trans. Sonics Ultrason., vol. SU-23, pp. 255-262, 1976.

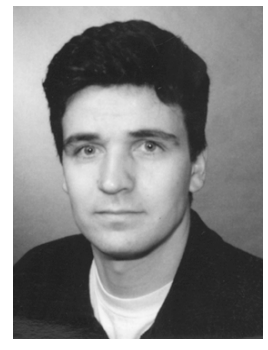

Bernhard Jakoby (S'91-A'95-SM'98) was born in Neuss, Germany, in 1966. He obtained the Dipl.-Ing. (M.Sc.) degree in Communication Engineering and the Ph.D. degree in Electrical Engineering from the Vienna University of Technology, Austria, in 1991 and 1994, respectively.

From 1991 to 1994 he worked as Research Assistant at the Institute of General Electrical Engineering and Electronics at the Vienna University of Technology, Austria. Subsequently, he obtained an Erwin Schrödinger grant from the Austrian Fund for Scientific Research (FWF) to perform research on the electrodynamics of complex media at the De- partment of Information Technology at the University of Ghent, Belgium. From 1996 to 1999 he held the position of a research associate and later assistant professor at the Electronic Instrumentation Laboratory of the Delft University of Technology, The Netherlands, working on the development of microacoustic sensors. Since 1999, he has been with the Robert Bosch GmbH, Reutlingen, Germany, performing research and development on sensors for automotive applications.

Bernhard Jakoby is a senior member of the IEEE. His research interests are focused on theory and applications in the fields of electromagnetics, microacoustics and integrated sensors.

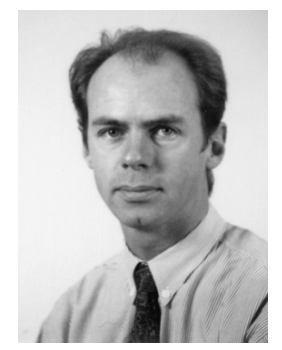

Michael J. Vellekoop was born in Amsterdam in 1960. He received the BSc degree in Physics from the HTS Dordrecht, The Netherlands, and the $\mathrm{PhD}$ degree in Electrical Engineering from the Delft University of Technology, The Netherlands, in 1982 and 1994, respectively.

From 1982 to 1984 , he stayed with the Royal Netherlands Naval College as a reserve officer after which he joined the Electronic Instrumentation Laboratory at the Delft University of Technology to work in the field of acoustic wave sensors. In addition, from 1988 to 1996, he was managing director of Xensor Integration B.V. and was involved in the development and the production of silicon sensors and actuators. Currently, he leads the Physical Chemosensors and Microacoustic Devices Group of the Electronic Instrumentation Laboratory, DIMES, where he holds the position of an associate professor. His recent research activities have been in the areas of microacoustic sensor systems for gas and liquid sensing applications, solid state sensor technology, and physical chemosensors. 